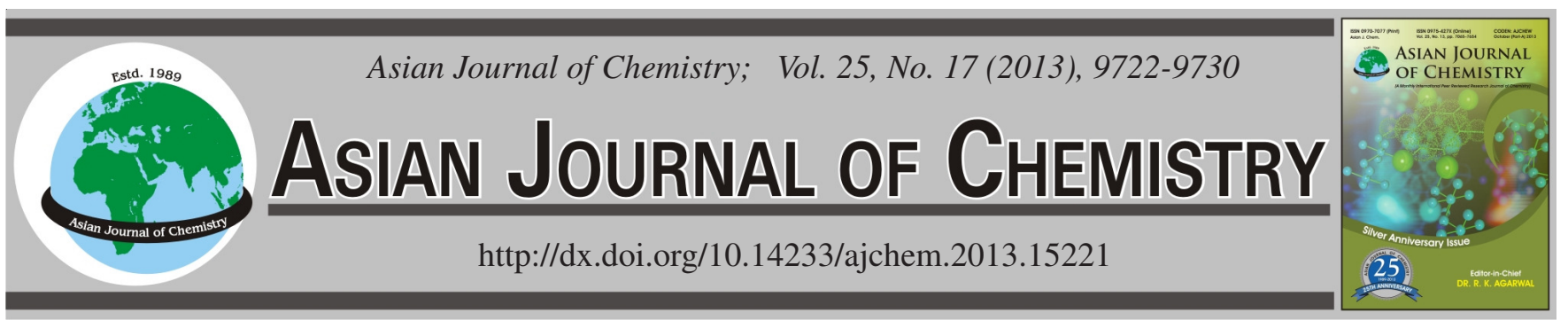

\title{
Synthesis and Quantum Chemical Calculations of 4-(2-Fluorophenyl)- 1-(2-oxoindolin-3-ylidene)thiosemicarbazone and its Zinc(II) Complex
}

\author{
F. KANDEMIRLI ${ }^{1, *}$, Y. AKKAYA ${ }^{2}$ and C.D. VuRdu ${ }^{1}$
}

${ }^{1}$ Biomedical Engineering Department, Faculty of Engineering and Architecture, Kastamonu University, Kastamonu, Turkey ${ }^{2}$ Department of Chemistry, Faculty of Science, Nigde University, Nigde, Turkey

*Corresponding author: Fax: +90 366 2154969; Tel: +90 366 2801908; E-mail: fkandemirli@yahoo.com

(Received: 28 January 2013;

Accepted: 18 October 2013)

AJC-14262

\begin{abstract}
In this study, wavenumbers, IR intensities and molecular parameters of 4-(2-fluorophenyl)-1-(2-oxoindolin-3-ylidene)thiosemicarbazone $\left(\mathrm{I}_{2} \mathrm{FPTH}_{2}\right)$ and its zinc(II) complex have been studied theoretically by using the $a b$ initio Hartree-Fock (HF) method with the 3-21G, 3$21 \mathrm{G}^{*}, 6-31 \mathrm{G}(\mathrm{d}, \mathrm{p}), 6-311 \mathrm{G}(\mathrm{d}, \mathrm{p}), 6-311++\mathrm{G}(\mathrm{d}, \mathrm{p})$ and 6-311++G $(2 \mathrm{~d}, 2 \mathrm{p})$ basis sets. In addition to theoretical study,they have been prepared for characterization of structure by means of elemental analyses which are FT-IR, electronic and ${ }^{1} \mathrm{H}$ NMR $\mathrm{Zn}$ (II) complex spectra. Moreover, according to the results of Fukui functions values of $\mathrm{I}_{2} \mathrm{FPTH}_{2}$, calculated with B3LYP/6-31G(d,p) and B3LYP/6$311 \mathrm{G}(\mathrm{d}, \mathrm{p})$, the contribution of sulphur to the HOMO is found predominant 47.69 and $49.16 \%$, respectively while the contribution of nitrogen to the LUMO is also found dominant as 24.82 and $24.21 \%$, respectively. The theoretical results of wavelengths, ${ }^{1} \mathrm{H}$ and ${ }^{13} \mathrm{C}$ NMR for wavenumbers are sensibly consistent with the results of experimental study.
\end{abstract}

Key Words: Thiosemicarbazone, DFT, ${ }^{1} \mathrm{H}$ NMR, ${ }^{13} \mathrm{C}$ NMR, FT-IR spectra.

\section{INTRODUCTION}

Isatin $(1 H$-indole-2,3-dione) is one of the endogenous compounds, which possess a wide range of biological activities. It has been also reported for derivatives of 4-(2-fluorophenyl)1-(2-oxoindolin-3-ylidene)thiosemicarbazone $\left(\mathrm{I}_{2} \mathrm{FPTH}_{2}\right)$ that results have been found considerable attention, because of variable bonding abilities and antiviral, antibacterial, antitumor and antifungal activity and inhibitory action ${ }^{1-9}$. Isatin thiosemicarbazones have been known to be certain of their coordination compounds.

Several isatin thiosemicarbazones and $\mathrm{Zn}$ (II) to $\mathrm{Ni}$ (II) complexes were previously studied theoretically and synthesized experimentally ${ }^{8,10-13}$. In the literature, the crystal structure of 4-(2-fluorophenyl)-1-(2-oxoindolin-3-ylidene)thiosemicarbazone $\left(\mathrm{I}_{2} \mathrm{FPTH}_{2}\right)$ were reported ${ }^{14}$, but there are no theoretical study about it and also no synthesis of $\mathrm{Zn}$ (II) complex of $\mathrm{I}_{2} \mathrm{FPTH}_{2}$ ligand.

According to results of the experimental and quantum chemical studies of 5-fluoroisatin-3-N(cyclohexylthiosemicarbazone) and its metal complexes, it was found that only one part of the ligand was coordinated to the metal ion resulting mono-nuclear complexes in $\mathrm{Ni}(\mathrm{II})$ and $\mathrm{Zn}$ (II) complexes ${ }^{15}$.

In this study, the vibrational spectrum, UV spectrum, ${ }^{13} \mathrm{C}$ NMR, ${ }^{1} \mathrm{H}$ NMR values, the molecular geometry, the atomic charges and molecular polarizability were carried out for 4-(2-fluorophenyl)-1-(2-oxoindolin-3-ylidene)thiosemicarbazone molecule by using density functional theory (DFT) with B3LYP hybrid functional and ab initio Hartree-Fock (HF) computations with the basis sets of $3-21 \mathrm{G}, 3-21 \mathrm{G}^{*}, 6-$ $31 \mathrm{G}(\mathrm{d}, \mathrm{p}), 6-311 \mathrm{G}(\mathrm{d}, \mathrm{p}), 6-311++\mathrm{G}(\mathrm{d}, \mathrm{p}), 6-311++\mathrm{G}(2 \mathrm{~d}, 2 \mathrm{p})^{16}$. Fukui functions were also calculated by using AOMix $\operatorname{program}^{17,18}$ from single-point calculations with B3LYP/6$311 \mathrm{G}(\mathrm{d}, \mathrm{p})^{16}$.

\section{EXPERIMENTAL}

4-(2-Fluorophenyl)-1-(2-oxoindolin-3-ylidene) thiosemicarbazone molecule was synthesized as reported ${ }^{18}$. The FTIR spectra was recorded using Jasco 300 FTIR spectrometer in the region 4000-200 $\mathrm{cm}^{-1}$. UV spectra was recorded UV-160A Shimadzu ${ }^{1}$.

Synthesis of bis[4-(2-fluorophenyl)thiosemicarbazonato)zinc(II) complex Zn[(I2FPTH $\left.)_{2}\right]$ : $1 \mathrm{mmol}(0.3142 \mathrm{~g})$ $\mathrm{I}_{2} \mathrm{FPTH}_{2}$ was dissolved in $20 \mathrm{~mL}$ of ethanol at $50-55^{\circ} \mathrm{C}$, before they was slowly added an ethanol solution $(10 \mathrm{~mL})$ of 0.5 mmol $(0.1097 \mathrm{~g})$ zinc acetate tetradehydrate. The mixture was refluxed for 6-9 h at a temperature of approximately. Light orange product was precipitated during the reaction and then it was washed with ethanol and diethyl ether. Finally, it was dried (m.p. $238{ }^{\circ} \mathrm{C}$ ). (calculated: (\%) C: 51.66, H: 2.92, N: 
15.91, S: 8.98 , found (\%): C: $51.206, \mathrm{H}: 2.97, \mathrm{~N}: 15.22$, S: 8.25).

Computational methodology: Theoretical calculations which are vibrational, wavenumbers, geometric parameters, atomic charges and the other molecular properties were performed by using the GAUSSIAN 09 package program ${ }^{16}$. Both ab initio calculations and DFT calculations were carried out by using B3LYP with basis sets of 3-21G, 3-21G*, 6$31 \mathrm{G}(\mathrm{d}, \mathrm{p}), 6-311 \mathrm{G}(\mathrm{d}, \mathrm{p}), 6-311++\mathrm{G}(\mathrm{d}, \mathrm{p}), 6-311++\mathrm{G}(2 \mathrm{~d}, 2 \mathrm{p})$. The harmonic unscaled vibrational frequencies were calculated for every each completely optimized geometry by analytical differentiation algorithms. Fukui functions were also calculated from results of single-point calculations with B3LYP/6$311 \mathrm{G}(\mathrm{d}, \mathrm{p})$ by using AOMix ${ }^{17,18}$.

\section{RESULTS AND DISCUSSION}

Geometric parameters: Crystallographic studies of 4-(2-fluorophenyl)-1-(2-oxoindolin-3-ylidene)thiosemicarbazone molecule show that molecules form inversion dimers in the crystal ${ }^{14}$. Therefore, the experimental X-ray data of 4-(2-fluorophenyl)-1-(2-oxoindolin-3-ylidene)thiosemicarbazone is used to calculate geometrical parameters. The conformation is stabilized by intramolecular N-H---N and $\mathrm{N}-\mathrm{H}---\mathrm{O}$ hydrogen bonds which generate $\mathrm{S}$ and the other $\mathrm{S}$ rings, respectively ${ }^{14}$. N-H---F and C-H---S interactions. The $\mathrm{X}$-ray structure of 4-(2-fluorophenyl)-1-(2-oxoindolin-3ylidene)-thiosemicarbazone dimer is shown in Fig. 1. The labeling of the atoms in the monomer form by using DFT method at the B3LYP/6-31G(d) level is also shown in Fig. 2.

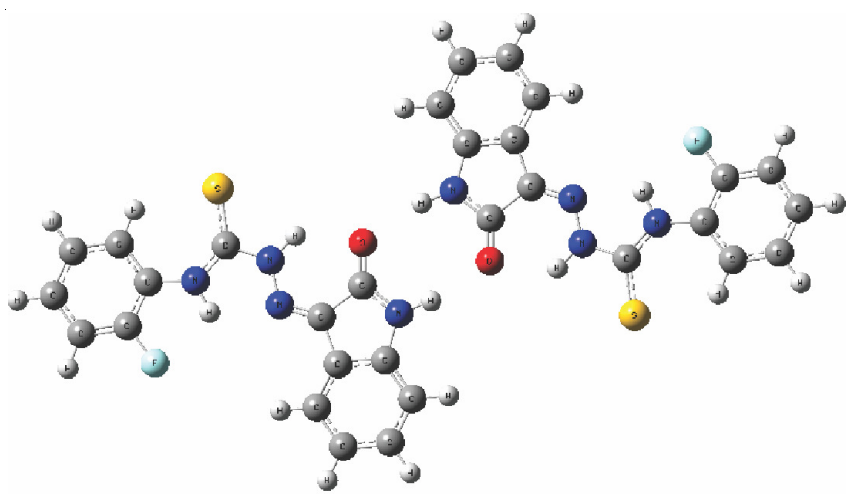

Fig. 1. Geometry of 4-(2-fluorophenyl)-1-(2-oxoindolin-3-ylidene) thiosemicarbazone dimer optimized at the B3LYP/6-311++G(2d,2p) level

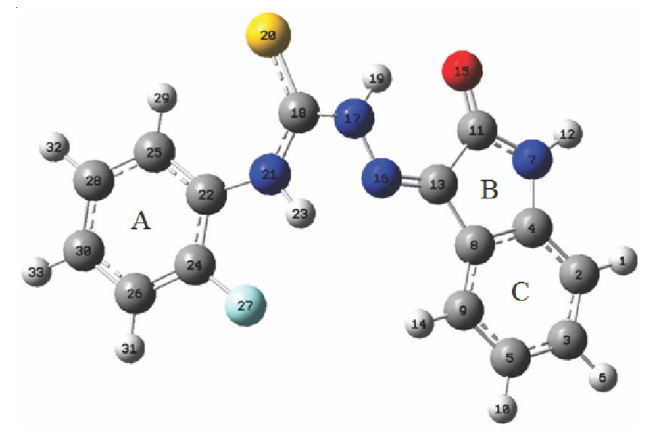

Fig. 2. Geometry of 4-(2-fluorophenyl)-1-(2-oxoindolin-3-ylidene)thiosemicarbazone calculated single point energy with the B3LYP/6$311++\mathrm{G}(2 \mathrm{~d}, 2 \mathrm{p})$ level
The optimized geometrical parameters, calculated by using the density functional and RHF methods, with the experimental data are summarized for studied molecule in Table-1. Calculated bond lengths are found systematically longer than experimental result. A statistical treatment of these data(see at the bottom of Table-1) shows that RHF for the bond lengths is slightly better than the B3LYP for all calculated basis sets. The highest correlation coefficient for bond lengths is 0.9745 for $R H F / 6-311++\mathrm{G}(2 \mathrm{~d}, 2 \mathrm{p})$.

The crystal structure of $\mathrm{I}^{\mathrm{FPTH}} \mathrm{H}_{2}$ was studied by Pervez et al. ${ }^{14}$. Experimental bond lengths of C28-C30, C30-C26, C26-C24 and C24-C22 belonging to phenyl group are1.351, $1.368,1.373$ and $1.370 \AA$ A while their theoretical bond lengths calculated by B3LYP/6-311++ G(2d,2p) are 1.389, 1.393, 1.376 and $1.400 \AA$, respectively. Moreover, experimental bond lengths of C18-S20, C18-N17, N17-N16, N16-C13 belonging to thiosemicarbazone group are $1.635,1.378,1.344$ and 1.291 $\AA$, respectively while their theoretical bond lengths calculated by B3LYP/6-311++ G(2d,2p) are 1.666, 1.390, 1.332 and $1.293 \AA$, respectively.

Some of the experimental and theoretical bond angles $\left({ }^{\circ}\right)$ values calculated by using B3LYP and RHF method for 4-(2fluorophenyl)-1-(2-oxoindolin-3-ylidene)thiosemicarbazone are summarized in Table-2. The correlation coefficients were calculated in $0.979,0.979,0.989,0.984,0.982,0.979$ and 0.990 for bond angles by using B3LYP.Besides, the correlation coefficients are $0.973,0.980,0.978,0.978,0.970,0.979$ and 0.979 for RHF with basis sets of $3-21 \mathrm{G}, 3-21 \mathrm{G}^{*}$, 6-31G(d,p), 6-311G(d,p), 6-311+G(d,p), 6-311++G(d,p) and $6-311++G(2 d, 2 p)$, respectively. The agreement between the calculated and the experimental bond angles is better than for the bond lengths.

The Mullikan and NBO charge values calculated with B3LYP and RHF methods are given in Table-3. Mullikan charges calculated with RHF/6-311G(d,p) predict a much more positive charge and negative charges on atoms for $\mathrm{I}_{2} \mathrm{FPTH}_{2}$, except C3 and C28 atoms, as seen in Table-3. It is resulted from NBO analysis that $\mathrm{O} 15$ atom has the largest negative charge in the indole ring and $\mathrm{N} 21$ has the largest negative charge in the thiosemicarbazone group.

Values of Fukui functions calculated with B3LYP/6$31 \mathrm{G}(\mathrm{d}, \mathrm{p})$ and B3LYP/6-311G(d,p) were summarized for $\mathrm{I}_{2} \mathrm{FPTH}_{2}$ in Table-4. The contributions of S20, N17 and N21 atoms to the HOMO are calculated with B3LYP/6-311G(d,p) as $47.69,6.74$ and $6.31 \%$, respectively, while they are calculated as $49.16,5.03$ and $8.35 \%$ by using B3LYP/6-31G(d,p), respectively. Moreover, the contributions of $\mathrm{C} 11, \mathrm{C} 13, \mathrm{O} 15$, $\mathrm{N} 16, \mathrm{~S} 20$ and N21 atoms to the LUMO are 9.88, 13.73, 8.27, $24.21,8.58$ and $1.72 \%$ in calculations done by B3LYP/6$311 \mathrm{G}(\mathrm{d}, \mathrm{p})$, respectively while they are $9.99,13.41,8.71,24.82$, 8.34 and $1.81 \%$ in calculations carried out by B3LYP/6$31 \mathrm{G}(\mathrm{d}, \mathrm{p})$, respectively.

IR studies: The IR spectra of solid $\mathrm{I}_{2} \mathrm{FPTH}_{2}$ and $\mathrm{Zn}\left[(\mathrm{I} 2 \mathrm{FPTH})_{2}\right]$ are shown in Fig. 3(a-c). The B3LYP/6$311 \mathrm{G}(\mathrm{d}, \mathrm{p})$ calculations were used to obtain computed harmonic vibrational frequencies and infrared intensities for $\mathrm{I}_{2} \mathrm{FPTH}_{2}$ and $\mathrm{Zn}\left[(\mathrm{I} 2 \mathrm{FPTH})_{2}\right]$. Table-5 lists theoretical and experimental bands of the IR spectra of the I2FPTH $\mathrm{H}_{2}$ and $\mathrm{Zn}\left[(\mathrm{I} 2 \mathrm{FPTH})_{2}\right]$ in the $4000-1250 \mathrm{~cm}^{-1}$ spectral region. 


\begin{tabular}{|c|c|c|c|c|c|c|c|c|}
\hline \multicolumn{9}{|c|}{$\begin{array}{l}\text { TABLE-1 } \\
\text { SELECTED EXPERIMENTAL AND THEORETICAL BOND LENGTHS (̊) THEORETICAL VALUES CALCULATED BY (A) } \\
\text { B3LYP AND (B) RHF METHOD FOR 4-(2-FLUOROPHENYL)-1-(2-OXOINDOLIN-3-YLIDENE)THIOSEMICARBAZONE }\end{array}$} \\
\hline \multicolumn{9}{|c|}{ (a) B3LYP } \\
\hline Atoms & Exp. & $3-21 G$ & $3-21 G^{*}$ & 6-31 G(d,p) & 6-311 G(d,p) & $6-311+G(d, p)$ & $6-311++\mathrm{G}(\mathrm{d}, \mathrm{p})$ & $6-311++G(2 d, 2 p)$ \\
\hline \multicolumn{9}{|c|}{ Bond lengths $(\AA)$} \\
\hline C28-C30 & 1.351 & 1.395 & 1.395 & 1.394 & 1.391 & 1.392 & 1.392 & 1.389 \\
\hline C26-C24 & 1.373 & 1.379 & 1.379 & 1.381 & 1.378 & 1.378 & 1.378 & 1.376 \\
\hline C24-F27 & 1.365 & 1.383 & 1.383 & 1.361 & 1.362 & 1.364 & 1.365 & 1.362 \\
\hline C24-C22 & 1.370 & 1.405 & 1.405 & 1.405 & 1.403 & 1.403 & 1.403 & 1.400 \\
\hline C22-N21 & 1.407 & 1.403 & 1.403 & 1.403 & 1.403 & 1.403 & 1.403 & 1.402 \\
\hline N21-C18 & 1.343 & 1.348 & 1.348 & 1.353 & 1.352 & 1.354 & 1.354 & 1.351 \\
\hline C18-S20 & 1.635 & 1.719 & 1.719 & 1.672 & 1.670 & 1.668 & 1.668 & 1.666 \\
\hline C18-N17 & 1.378 & 1.390 & 1.390 & 1.393 & 1.393 & 1.394 & 1.394 & 1.390 \\
\hline N16-C13 & 1.291 & 1.301 & 1.301 & 1.299 & 1.295 & 1.295 & 1.295 & 1.293 \\
\hline C13-C11 & 1.498 & 1.501 & 1.501 & 1.500 & 1.502 & 1.501 & 1.501 & 1.500 \\
\hline C11-O15 & 1.229 & 1.259 & 1.259 & 1.240 & 1.233 & 1.235 & 1.235 & 1.234 \\
\hline C11-N7 & 1.348 & 1.370 & 1.370 & 1.365 & 1.363 & 1.363 & 1.363 & 1.362 \\
\hline N7-C4 & 1.406 & 1.413 & 1.413 & 1.408 & 1.407 & 1.407 & 1.407 & 1.406 \\
\hline $\mathrm{C} 4-\mathrm{C} 2$ & 1.367 & 1.385 & 1.385 & 1.387 & 1.385 & 1.385 & 1.385 & 1.382 \\
\hline $\mathrm{C} 2-\mathrm{C} 3$ & 1.376 & 1.403 & 1.403 & 1.401 & 1.399 & 1.399 & 1.399 & 1.397 \\
\hline C3-C5 & 1.377 & 1.400 & 1.400 & 1.399 & 1.397 & 1.398 & 1.397 & 1.395 \\
\hline C5-C9 & 1.377 & 1.400 & 1.400 & 1.398 & 1.395 & 1.396 & 1.396 & 1.393 \\
\hline C9-C8 & 1.387 & 1.390 & 1.390 & 1.393 & 1.391 & 1.391 & 1.391 & 1.389 \\
\hline $\mathrm{C} 8-\mathrm{C} 4$ & 1.381 & 1.416 & 1.416 & 1.412 & 1.409 & 1.409 & 1.409 & 1.407 \\
\hline C13-C8 & 1.445 & 1.455 & 1.455 & 1.456 & 1.455 & 1.456 & 1.456 & 1.454 \\
\hline C22-C25 & 1.375 & 1.399 & 1.399 & 1.401 & 1.399 & 1.400 & 1.400 & 1.397 \\
\hline C25-C28 & 1.377 & 1.397 & 1.397 & 1.397 & 1.394 & 1.394 & 1.394 & 1.391 \\
\hline $\mathrm{R}^{2}$ & & 0.9567 & 0.9567 & 0.9684 & 0.9721 & 0.9724 & 0.9724 & 0.9738 \\
\hline C30-C26 & 1.368 & 1.398 & 1.387 & 1.386 & 1.386 & 1.386 & 1.383 & 1.393 \\
\hline C26-C24 & 1.373 & 1.378 & 1.370 & 1.369 & 1.369 & 1.369 & 1.367 & 1.376 \\
\hline C24-F27 & 1.365 & 1.383 & 1.341 & 1.337 & 1.336 & 1.336 & 1.334 & 1.362 \\
\hline C24-C22 & 1.370 & 1.406 & 1.391 & 1.390 & 1.390 & 1.390 & 1.387 & 1.400 \\
\hline C22-N21 & 1.407 & 1.401 & 1.408 & 1.408 & 1.408 & 1.408 & 1.406 & 1.402 \\
\hline N21-C18 & 1.343 & 1.355 & 1.334 & 1.335 & 1.336 & 1.336 & 1.333 & 1.351 \\
\hline C18-S20 & 1.635 & 1.668 & 1.675 & 1.673 & 1.671 & 1.671 & 1.670 & 1.666 \\
\hline C18-N17 & 1.378 & 1.401 & 1.365 & 1.364 & 1.365 & 1.365 & 1.361 & 1.390 \\
\hline N17-N16 & 1.344 & 1.373 & 1.336 & 1.335 & 1.335 & 1.335 & 1.334 & 1.332 \\
\hline N16-C13 & 1.291 & 1.301 & 1.260 & 1.258 & 1.258 & 1.258 & 1.255 & 1.293 \\
\hline C13-C11 & 1.498 & 1.500 & 1.511 & 1.514 & 1.514 & 1.514 & 1.512 & 1.500 \\
\hline C11-O15 & 1.229 & 1.260 & 1.207 & 1.201 & 1.202 & 1.202 & 1.202 & 1.234 \\
\hline C11-N7 & 1.348 & 1.370 & 1.345 & 1.345 & 1.345 & 1.345 & 1.342 & 1.362 \\
\hline N7-C4 & 1.406 & 1.413 & 1.403 & 1.403 & 1.403 & 1.403 & 1.402 & 1.406 \\
\hline $\mathrm{C} 4-\mathrm{C} 2$ & 1.367 & 1.385 & 1.376 & 1.375 & 1.376 & 1.376 & 1.372 & 1.382 \\
\hline $\mathrm{C} 2-\mathrm{C} 3$ & 1.376 & 1.403 & 1.391 & 1.390 & 1.391 & 1.391 & 1.388 & 1.397 \\
\hline C3-C5 & 1.377 & 1.400 & 1.388 & 1.387 & 1.388 & 1.388 & 1.385 & 1.395 \\
\hline C5-C9 & 1.377 & 1.400 & 1.388 & 1.388 & 1.388 & 1.389 & 1.386 & 1.393 \\
\hline C9-C8 & 1.387 & 1.390 & 1.380 & 1.379 & 1.379 & 1.379 & 1.376 & 1.389 \\
\hline C8-C4 & 1.381 & 1.416 & 1.393 & 1.391 & 1.391 & 1.391 & 1.388 & 1.407 \\
\hline C13-C8 & 1.445 & 1.455 & 1.466 & 1.466 & 1.467 & 1.467 & 1.465 & 1.454 \\
\hline $\mathrm{C} 22-\mathrm{C} 25$ & 1.375 & 1.399 & 1.387 & 1.386 & 1.387 & 1.387 & 1.384 & 1.397 \\
\hline C25-C28 & 1.377 & 1.397 & 1.388 & 1.388 & 1.388 & 1.388 & 1.385 & 1.391 \\
\hline $\mathrm{R}^{2}$ & & 0.964 & 0.971 & 0.9741 & 0.9744 & 0.9737 & 0.9729 & 0.9745 \\
\hline
\end{tabular}

The IR spectrum of $\mathrm{I}_{2} \mathrm{FPTH}_{2}$ shows that bands at 3313 $\mathrm{cm}^{-1}$ attributed to $v(\mathrm{NH}) \mathrm{cm}^{-1}$ vibration of the indole ring and thiosemicarbazone moiety. On complex formation, the position of these bands shifted to $3364 \mathrm{~cm}^{-1}$ due to changes in hydrogen bonding. A strong band at 1690 and $1697 \mathrm{~cm}^{-1}$ assigned to $(\mathrm{C}=\mathrm{O})$ stretching vibration of ligand and its $\mathrm{Zn}(\mathrm{II})$ complex, respectively. This indicates that $\mathrm{CO}$ group is uncoordinated in its $\mathrm{Zn}(\mathrm{II})$ complex.
Abdullah et al..$^{20}$ reported that all the thiosemicarbazones remain in their thione form due to the existence of a strong band in the region 1038-1026 $\mathrm{cm}^{-1}$ due to $v(\mathrm{C}=\mathrm{S})$ and absence of any band in the region $2600-2500 \mathrm{~cm}^{-1}$ due to $v(\mathrm{C}-\mathrm{SH})$ for steroidal thiosemicarbazones.

The $\mathrm{C}=\mathrm{S}$ band appearing at $817 \mathrm{~cm}^{-1}$ in the IR spectrum of $\mathrm{I}_{2} \mathrm{FPTH}_{2}$ ligand is shifted towards lower wave number in 


\begin{tabular}{|c|c|c|c|c|c|c|c|c|}
\hline \multicolumn{9}{|c|}{$\begin{array}{c}\text { TABLE-2 } \\
\text { SELECTED EXPERIMENTAL AND THEORETICAL BOND ANGLES }\left({ }^{\circ}\right) \text { VALUES } \\
\text { CALCULATED BY (A) B3LYP AND (b) RHF METHOD FOR I2FPTH }{ }_{2} \\
\end{array}$} \\
\hline \multicolumn{9}{|c|}{ (a) B3LYP } \\
\hline Atoms & Exp. & $3-21 \mathrm{G}$ & $3-21 G^{*}$ & $6-31 \mathrm{G}(\mathrm{d}, \mathrm{p})$ & 6-311 G(d,p) & $6-311+\mathrm{G}(\mathrm{d}, \mathrm{p})$ & $6-311++\mathrm{G}(\mathrm{d}, \mathrm{p})$ & $6-311++\mathrm{G}(2 \mathrm{~d}, 2 \mathrm{p})$ \\
\hline \multicolumn{9}{|c|}{ Bond angles $\left({ }^{\circ} \mathrm{C}\right)$} \\
\hline C1-C3-F32 & 120.1 & 120.7 & 120.7 & 119.5 & 119.3 & 119.1 & 119.1 & 119.1 \\
\hline F32-C3-C13 & 116.5 & 116.9 & 116.9 & 117.3 & 119.3 & 117.5 & 117.5 & 117.5 \\
\hline C3-C13-N19 & 116.8 & 114.7 & 114.7 & 115.4 & 115.7 & 117.5 & 116.0 & 116.0 \\
\hline C14-C13-N19 & 126.6 & 127.2 & 127.2 & 127.1 & 126.9 & 126.8 & 126.8 & 126.8 \\
\hline C13-C3-C1 & 123.4 & 122.4 & 122.4 & 123.1 & 123.1 & 123.4 & 123.4 & 123.4 \\
\hline $\mathrm{C} 3-\mathrm{C} 1-\mathrm{C} 2$ & 118.1 & 119.1 & 119.1 & 118.6 & 118.7 & 118.6 & 118.6 & 119.5 \\
\hline $\mathrm{C} 1-\mathrm{C} 2-\mathrm{C} 8$ & 120.2 & 119.5 & 119.5 & 119.6 & 119.5 & 119.5 & 119.5 & 121.3 \\
\hline C2-C8-C14 & 120.8 & 121.1 & 121.1 & 121.3 & 121.3 & 121.3 & 121.3 & 120.1 \\
\hline C8-C14-C13 & 120.8 & 119.8 & 119.8 & 119.8 & 120.0 & 120.1 & 120.1 & 117.2 \\
\hline C14-C13-C3 & 116.6 & 118.1 & 118.1 & 117.6 & 117.4 & 117.2 & 117.2 & 117.2 \\
\hline C13-N19-C16 & 130.4 & 132.0 & 132.0 & 132.3 & 132.3 & 132.1 & 132.2 & 132.1 \\
\hline N19-C16-S7 & 129.5 & 130.4 & 130.4 & 130.0 & 129.9 & 129.9 & 129.9 & 129.9 \\
\hline N19-C16-N20 & 112.3 & 112.4 & 112.4 & 112.5 & 112.4 & 112.5 & 112.7 & 112.5 \\
\hline S7-C16-N20 & 118.2 & 117.2 & 117.2 & 117.5 & 117.6 & 117.5 & 117.4 & 117.5 \\
\hline C16-N20-N21 & 121.1 & 119.3 & 119.3 & 121.3 & 121.4 & 117.8 & 121.7 & 121.5 \\
\hline N20-N21-C18 & 117.8 & 117.8 & 117.8 & 119.1 & 119.5 & 119.4 & 119.4 & 119.4 \\
\hline N21-C18-C12 & 127.3 & 125.9 & 125.9 & 126.7 & 126.7 & 126.8 & 126.8 & 126.8 \\
\hline N21-C18-C15 & 126.0 & 127.5 & 127.5 & 126.6 & 126.6 & 126.6 & 126.7 & 126.6 \\
\hline C18-C12-O9 & 127.1 & 125.6 & 125.6 & 126.4 & 126.5 & 126.5 & 126.6 & 126.5 \\
\hline O9-C12-N6 & 127.2 & 127.8 & 127.8 & 127.4 & 127.4 & 127.3 & 127.2 & 127.3 \\
\hline N6-C10-C4 & 128.9 & 129.5 & 129.5 & 128.8 & 128.8 & 128.8 & 128.7 & 128.8 \\
\hline C17-C15-C18 & 133.3 & 132.7 & 132.7 & 133.4 & 133.3 & 133.4 & 133.4 & 133.4 \\
\hline C15-C18-C12 & 106.6 & 106.5 & 106.5 & 106.7 & 106.6 & 106.6 & 106.5 & 106.6 \\
\hline C18-C12-N6 & 105.8 & 106.5 & 106.5 & 106.2 & 106.1 & 106.2 & 106.2 & 106.2 \\
\hline C12-N6-C10 & 111.8 & 110.9 & 110.9 & 111.3 & 111.4 & 111.3 & 111.3 & 111.3 \\
\hline N6-C10-C15 & 108.9 & 109.3 & 109.3 & 109.4 & 109.4 & 109.3 & 109.4 & 109.3 \\
\hline C10-C15-C17 & 119.8 & 120.6 & 120.6 & 120.1 & 120.1 & 120.0 & 120.0 & 120.0 \\
\hline C15-C17-C11 & 117.9 & 118.5 & 118.5 & 118.6 & 118.6 & 118.6 & 118.6 & 118.6 \\
\hline C17-C11-C5 & 121.1 & 120.5 & 120.5 & 120.6 & 120.6 & 120.6 & 120.6 & 120.6 \\
\hline $\mathrm{C} 11-\mathrm{C} 5-\mathrm{C} 4$ & 121.6 & 121.4 & 121.4 & 121.5 & 121.4 & 121.4 & 121.4 & 121.4 \\
\hline C5-C4-C10 & 117.4 & 117.8 & 117.8 & 117.4 & 117.4 & 117.4 & 117.4 & 117.4 \\
\hline C4-C10-C15 & 122.4 & 121.2 & 121.2 & 121.9 & 121.8 & 121.9 & 121.9 & 121.9 \\
\hline $\mathrm{R}^{2}$ & & 0.979 & 0.979 & 0.989 & 0.984 & 0.982 & 0.979 & 0.990 \\
\hline \multicolumn{9}{|c|}{ (b) RHF } \\
\hline C1-C3-F32 & 120.1 & 120.1 & 120.8 & 120.0 & 119.0 & 120.9 & 120.9 & 118.9 \\
\hline F32-C3-C13 & 116.5 & 116.0 & 120.7 & 117.6 & 117.8 & 117.8 & 117.8 & 117.8 \\
\hline C3-C13-N19 & 116.8 & 116.6 & 127.3 & 115.0 & 115.2 & 116.3 & 115.3 & 115.3 \\
\hline C14-C13-N19 & 126.6 & 124.6 & 114.7 & 127.5 & 127.4 & 127.4 & 127.4 & 127.4 \\
\hline C13-C3-C1 & 123.4 & 122.9 & 122.5 & 123.3 & 123.2 & 123.4 & 123.4 & 123.3 \\
\hline $\mathrm{C} 3-\mathrm{C} 1-\mathrm{C} 2$ & 118.1 & 118.9 & 119.1 & 118.6 & 118.7 & 118.7 & 118.7 & 118.7 \\
\hline $\mathrm{C} 1-\mathrm{C} 2-\mathrm{C} 8$ & 120.2 & 119.4 & 119.5 & 119.4 & 119.4 & 120.3 & 119.3 & 119.4 \\
\hline C2-C8-C14 & 120.8 & 121.1 & 121.1 & 121.3 & 121.3 & 121.3 & 121.3 & 121.3 \\
\hline C8-C14-C13 & 120.8 & 119.8 & 119.9 & 119.9 & 120.0 & 120.0 & 120.0 & 120.1 \\
\hline C14-C13-C3 & 116.6 & 117.8 & 118.0 & 117.4 & 117.4 & 117.3 & 117.3 & 117.3 \\
\hline C13-N19-C16 & 130.4 & 132.2 & 131.9 & 132.6 & 132.6 & 130.5 & 132.5 & 132.6 \\
\hline N19-C16-S7 & 129.5 & 129.6 & 130.5 & 129.2 & 129.1 & 129.1 & 129.1 & 129.1 \\
\hline N19-C16-N20 & 112.3 & 114.0 & 111.5 & 113.8 & 113.8 & 112.8 & 113.8 & 114.0 \\
\hline S7-C16-N20 & 118.2 & 116.4 & 118.0 & 117.0 & 117.1 & 117.1 & 117.1 & 116.9 \\
\hline C16-N20-N21 & 121.1 & 119.5 & 119.5 & 121.3 & 121.4 & 121.5 & 121.5 & 121.6 \\
\hline N20-N21-C18 & 117.8 & 120.1 & 118.1 & 120.7 & 120.8 & 117.7 & 120.7 & 120.7 \\
\hline N21-C18-C12 & 127.3 & 126.8 & 125.9 & 127.6 & 127.7 & 120.7 & 127.7 & 127.7 \\
\hline N21-C18-C15 & 126.0 & 127.5 & 127.5 & 126.7 & 126.7 & 126.7 & 126.7 & 126.8 \\
\hline $\mathrm{C} 18-\mathrm{C} 12-\mathrm{O} 9$ & 127.1 & 125.7 & 125.7 & 126.3 & 126.4 & 127.4 & 126.4 & 126.6 \\
\hline O9-C12-N6 & 127.2 & 127.9 & 127.8 & 127.5 & 127.5 & 127.4 & 127.4 & 127.2 \\
\hline N6-C10-C4 & 128.9 & 129.2 & 129.5 & 128.4 & 128.4 & 128.4 & 128.4 & 128.3 \\
\hline C17-C15-C18 & 133.3 & 132.1 & 132.7 & 132.9 & 132.8 & 133.4 & 132.9 & 132.9 \\
\hline C15-C18-C12 & 106.6 & 105.7 & 106.6 & 105.7 & 105.7 & 105.6 & 105.6 & 105.6 \\
\hline C18-C12-N6 & 105.8 & 106.4 & 106.5 & 106.2 & 106.1 & 105.8 & 106.1 & 106.2 \\
\hline C12-N6-C10 & 111.8 & 111.5 & 110.9 & 111.8 & 111.9 & 111.9 & 111.9 & 111.8 \\
\hline N6-C10-C15 & 108.9 & 109.4 & 109.2 & 109.7 & 109.7 & 108.7 & 109.7 & 109.8 \\
\hline
\end{tabular}




\begin{tabular}{cccccccc}
\hline C10-C15-C17 & 119.8 & 120.9 & 120.6 & 120.5 & 120.5 & 119.9 & 120.5 \\
C15-C17-C11 & 117.9 & 118.4 & 118.5 & 118.4 & 118.5 & 118.5 & 118.5 \\
C17-C11-C5 & 121.1 & 120.3 & 120.5 & 120.4 & 120.3 & 121.1 & 120.3 \\
C11-C5-C4 & 121.6 & 121.5 & 121.4 & 121.7 & 121.7 & 121.6 & 120.5 \\
C5-C4-C10 & 117.4 & 117.6 & 117.8 & 117.2 & 117.2 & 117.5 & 121.6 \\
C4-C10-C15 & 122.4 & 121.4 & 121.2 & 121.9 & 121.8 & 122.4 & 11.6 \\
R $^{2}$ & & 0.973 & 0.980 & 0.978 & 0.978 & 0.970 & 121.9 \\
\hline
\end{tabular}

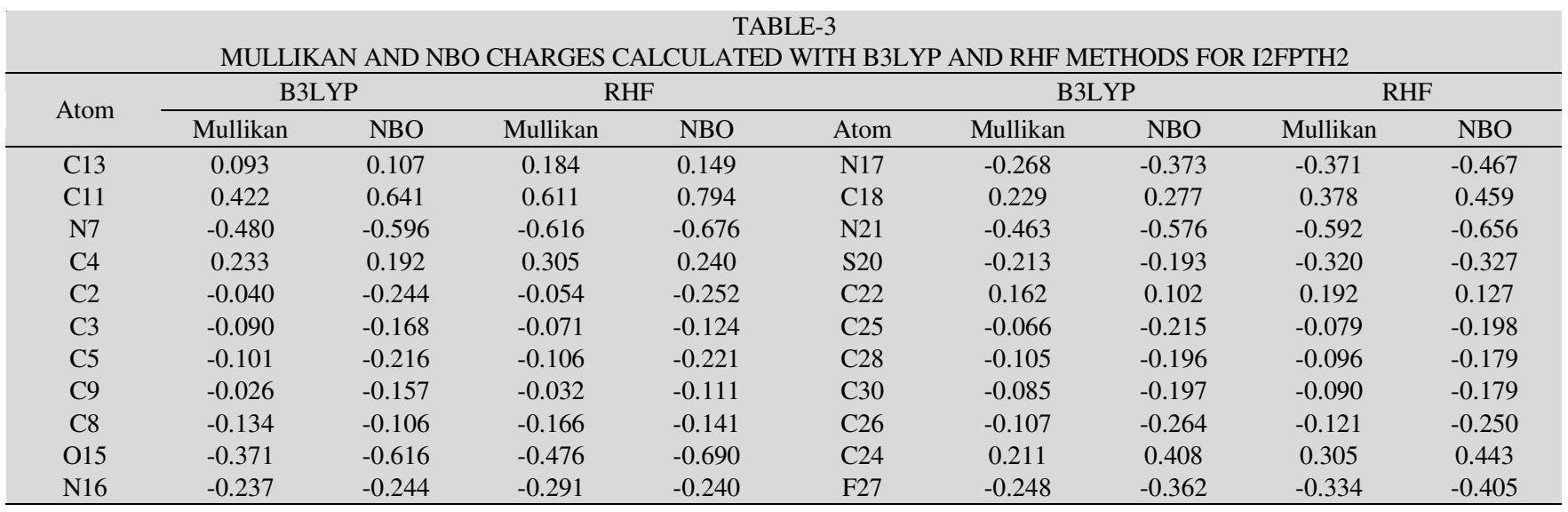

TABLE-4

FUKUI FUNCTIONS VALUES FOR I2FPTH ${ }_{2}$

\begin{tabular}{|c|c|c|c|c|c|}
\hline \multicolumn{3}{|c|}{ HOMO } & \multicolumn{3}{|c|}{ LUMO } \\
\hline Atoms & $6-31 G(d, p)$ & 6-311G (d,p) & Atoms & $6-31 G(d, p)$ & 6-311G (d,p) \\
\hline C3 & 3.33 & 4.29 & $\mathrm{C} 3$ & 6.46 & 6.68 \\
\hline $\mathrm{C} 4$ & 2.66 & 3.47 & $\mathrm{C} 4$ & 3.94 & 3.88 \\
\hline $\mathrm{C} 5$ & 1.28 & 1.78 & N7 & 1.41 & 1.33 \\
\hline $\mathrm{C} 8$ & 2.62 & 3.53 & $\mathrm{C} 8$ & 3.15 & 3.42 \\
\hline C9 & 1.18 & 1.45 & C9 & 4.99 & 5.24 \\
\hline $\mathrm{C} 13$ & 3.93 & 4.92 & $\mathrm{C} 11$ & 9.99 & 9.88 \\
\hline $\mathrm{O} 15$ & 2.36 & 2.94 & $\mathrm{C} 13$ & 13.41 & 13.73 \\
\hline N16 & 1.2 & 1.59 & $\mathrm{O} 15$ & 8.71 & 8.27 \\
\hline N17 & 5.03 & 6.74 & N16 & 24.82 & 24.21 \\
\hline $\mathrm{C} 18$ & 1.16 & 1.71 & N17 & 4.1 & 3.84 \\
\hline S20 & 49.16 & 47.69 & $\mathrm{C} 18$ & 5.84 & 5.84 \\
\hline $\mathrm{N} 21$ & 8.35 & 6.31 & $\mathrm{~S} 20$ & 8.34 & 8.58 \\
\hline $\mathrm{C} 22$ & 3.02 & 2 & $\mathrm{~N} 21$ & 1.81 & 1.72 \\
\hline $\mathrm{C} 24$ & 3.77 & 2.73 & - & - & - \\
\hline $\mathrm{C} 28$ & 1.95 & 1.46 & - & - & - \\
\hline $\mathrm{C} 30$ & 4.97 & 3.49 & - & - & - \\
\hline
\end{tabular}

the spectra of $\mathrm{Zn}\left[(\mathrm{I} 2 \mathrm{FPTH})_{2}\right]$ complex. This is due to coordination of thione sulphur to the zinc ion. The strong band appearing at $1571 \mathrm{~cm}^{-1}$ in the IR spectrum of $\mathrm{I}_{2} \mathrm{FPTH}_{2}$ ligand shifted towards lower wave number in the spectra of $\mathrm{Zn}\left[(\mathrm{I} 2 \mathrm{FPTH})_{2}\right]$ complex. Shifting towards lower wave number is due to the coordination of azomethine nitrogen with metal ion.

NMR studies: Chemical shifts of the ${ }^{1} \mathrm{H}$ and ${ }^{13} \mathrm{C}$ NMR for $\mathrm{I}_{2} \mathrm{FPTH}_{2}$ are given in Tables 6 and 7, respectively. These chemical shifts were obtained from calculations with RHF and DFT methods employing the basis sets 3-21G, 3-21G*, 6-31G(d,p), 6-311G(d,p), 6-311+G(d,p), 6-311++G(d,p) and $6-311++G(2 d, 2 p) .{ }^{1} \mathrm{H}$ NMR chemical shifts correlation coefficient for $\mathrm{I}_{2} \mathrm{FPTH}_{2}$ except indole hydrogen employing the basis sets 3-21G, 3-21G*, 6-31G(d,p), 6-311G(d,p), 6$311+\mathrm{G}(\mathrm{d}, \mathrm{p}), 6-311++\mathrm{G}(\mathrm{d}, \mathrm{p})$ and $6-311++\mathrm{G}(2 \mathrm{~d}, 2 \mathrm{p})$ are 0.654 , $0.864,0.654,0.639,0.676,0.684$ and 0.693 for RHF method, respectively while they are $0.852,0.854,0.831,0.808,0.825$, 0.830 and 0.830 for B3LYP method, respectively. H30 and $\mathrm{H} 25$ chemical shifts are assigned experimentally at 11.26 , 12.86 and theoretically calculated as $9.24,11.41$ by using B3LYP/6-311+G(d,p). In addition, ${ }^{13} \mathrm{C}$ NMR chemical shifts correlation coefficient for $\mathrm{I}_{2} \mathrm{FPTH}_{2}$ employing the basis sets 3-21G, 3-21G*, 6-31G(d,p), 6-311G(d,p), 6-311+G(d,p), 6-311++G(d,p) and 6-311++G(2d,2p) are 0.872, 0.967, 0.947, 0.946, 0.946, 0.946 and 0.953 , respectively, obtained from RHF method and $0.872,0.967,0.947,0.970,0.970,0.968$ and 0.969 , respectively, obtained from B3LYP method. The NMR chemical shifts employing the basis sets of 6-311G(d,p) and $6-311+\mathrm{G}(\mathrm{d}, \mathrm{p})$ are given in Table- 8 together with experimental values for $\mathrm{Zn}\left[(\mathrm{I} 2 \mathrm{FPTH})_{2}\right]$.

Proton NMR of $\mathrm{Zn}\left[(\mathrm{I} 2 \mathrm{FPTH})_{2}\right]$ shows a characteristic indole $\mathrm{NH}$ signal at $\delta 10.67 \mathrm{ppm}$ that is similar to that for the $\mathrm{I}_{2} \mathrm{FPTH}_{2}$ ligand at $\delta 10.71 \mathrm{ppm}$. The characteristic proton signals 


\begin{tabular}{|c|c|c|c|c|c|c|}
\hline \multicolumn{7}{|c|}{$\begin{array}{l}\text { TABLE-5 } \\
\text { EXPERIMENTAL AND THEORETICAL FT-IR VALUES OF 4-(2-FLUOROPHENYL)-1-(2-OXOINDOLIN-3- } \\
\left.\text { YLIDENE)THIOSEMICARBAZONE “I2FPTH" AND ITS ZINC(II) COMPLEX“ZZ[(I2FPTH })_{2}\right] "\end{array}$} \\
\hline \multicolumn{3}{|c|}{ Ligand } & \multicolumn{4}{|c|}{$\mathrm{Zn}$} \\
\hline Exp. & 6-311 & Intensity & Exp. & $6-311$ & Intensity & Signs \\
\hline 3313 & 3535 & 76 & 3364 & 3647 & 73 & $v(\mathrm{~N} 7 \mathrm{H} 12)$ \\
\hline 3187 & 3492 & 116 & 3180 & 3570 & 218 & $v(\mathrm{~N} 21 \mathrm{H})$ \\
\hline 3152 & 3438 & 92 & - & - & - & $v(\mathrm{~N} 17 \mathrm{H})$ \\
\hline 3073 & 3237 & 10 & 3063 & 3239 & 138 & $v(\mathrm{C} 25 \mathrm{H})$ \\
\hline- & 3199 & 15 & - & - & - & $v(\mathrm{CH})$ ring $\mathrm{C}$ \\
\hline- & 3193 & 14 & - & 3204 & 20 & $v(\mathrm{CH})$ ring $\mathrm{A}$ \\
\hline- & 3191 & 15 & - & 3199 & 22 & $v(\mathrm{CH})$ ring $\mathrm{C}$ \\
\hline- & - & - & - & 3187 & 14 & $v(\mathrm{CH})$ ring $\mathrm{C}$ \\
\hline 1690 & 1731 & 274 & 1697 & 1793 & 577 & $v(\mathrm{C} 11 \mathrm{O} 15), v(\mathrm{C} 13 \mathrm{~N} 16), \delta(\mathrm{N} 17 \mathrm{H} 19), \delta(\mathrm{N} 7 \mathrm{H} 12)$ \\
\hline 1618 & 1663 & 82 & 1611 & 1662 & 203 & $\delta(\mathrm{N} 7 \mathrm{H}), v(\mathrm{CC})$ ring $\mathrm{C}$ \\
\hline & 1659 & 130 & 1595 & 1635 & 81 & $\delta(\mathrm{N} 21 \mathrm{H} 23), v(\mathrm{CC})$ ring $\mathrm{A}, \delta(\mathrm{CH})$ ring $\mathrm{A}$ \\
\hline 1594 & 1639 & 135 & - & - & - & $v(\mathrm{C} 18 \mathrm{~N} 21), \delta(\mathrm{N} 21 \mathrm{H}), v(\mathrm{CH})$ ring A \\
\hline 1571 & 1625 & 150 & - & - & - & $\delta(\mathrm{N} 17 \mathrm{H}), v(\mathrm{C} 13 \mathrm{~N} 16), \delta(\mathrm{N} 7 \mathrm{H})$ \\
\hline 1538 & 1590 & 737 & 1505 & 1595 & 75 & $\delta(\mathrm{N} 21 \mathrm{H}), v(\mathrm{C} 18 \mathrm{~N} 21), \delta(\mathrm{CH})$ ring A \\
\hline 1530 & 1528 & 108 & - & - & - & $\delta(\mathrm{N} 17 \mathrm{H}), \delta(\mathrm{N} 21 \mathrm{H}), v(\mathrm{C} 18 \mathrm{~S} 20), v(\mathrm{CH})$ ring A \\
\hline- & - & - & 1456 & 1575 & 819 & $\delta(\mathrm{N} 21 \mathrm{H}), v(\mathrm{C} 18 \mathrm{~N} 21), \delta(\mathrm{CH})$ ring A \\
\hline 1470 & 1518 & 39 & - & - & - & $\delta(\mathrm{N} 7 \mathrm{H}), \delta(\mathrm{N} 21 \mathrm{H}), v(\mathrm{C} 18 \mathrm{~S} 20), v(\mathrm{CH})$ ring $\mathrm{A}, v(\mathrm{CH})$ ring $\mathrm{C}$ \\
\hline- & - & - & 1418 & 1514 & 78 & $\delta(\mathrm{N} 21 \mathrm{H}), \delta(\mathrm{CH})$ ring $\mathrm{A}$ \\
\hline- & - & - & - & 1499 & 136 & $\delta(\mathrm{CH})$ ring $\mathrm{C}$ \\
\hline 1463 & 1508 & 77 & - & - & - & $\delta(\mathrm{N} 17 \mathrm{H}), \delta(\mathrm{N} 21 \mathrm{H}), \delta(\mathrm{CH})$ ring $\mathrm{A}, \delta(\mathrm{CH})$ ring $\mathrm{C}, v(\mathrm{C} 18 \mathrm{~S} 20)$ \\
\hline 1451 & 1495 & 60 & - & - & - & $\delta(\mathrm{N} 17 \mathrm{H}), \delta(\mathrm{CH})$ ring $\mathrm{A}, \delta(\mathrm{CH})$ ring $\mathrm{C}$ \\
\hline 1407 & 1488 & 183 & - & - & - & $\delta(\mathrm{N} 17 \mathrm{H}), \delta(\mathrm{CH})$ ring $\mathrm{A}, \delta(\mathrm{CH})$ ring $\mathrm{C}, v(\mathrm{C} 18 \mathrm{~N} 21)$ \\
\hline- & 1425 & 81 & - & - & - & $\delta(\mathrm{N} 7 \mathrm{H}), v(\mathrm{C} 18 \mathrm{~N} 21), \delta(\mathrm{CH})$ ring $\mathrm{C}$ \\
\hline- & - & - & 1383 & 1444 & 1287 & $v(\mathrm{~N} 21 \mathrm{C} 18 \mathrm{~N} 17), v(\mathrm{~N} 16 \mathrm{C} 13)$ \\
\hline 1344 & 1400 & 324 & & 1441 & 2159 & $\delta(\mathrm{N} 21 \mathrm{H}), \delta(\mathrm{N} 17 \mathrm{H}), \delta(\mathrm{N} 7 \mathrm{H}), v(\mathrm{C} 18 \mathrm{~N} 21)$ \\
\hline- & - & - & 1336 & 1420 & 157 & $\delta(\mathrm{N} 7 \mathrm{H}), \delta(\mathrm{CH})$ ring $\mathrm{C}$ \\
\hline 1320 & 1366 & 53 & - & - & - & $\delta(\mathrm{N} 7 \mathrm{H}), \delta(\mathrm{CH})$ ring $\mathrm{C}$ \\
\hline 1296 & 1349 & 34 & 1293 & 1363 & 194 & $v(\mathrm{CC})$ ring $\mathrm{A}, \mathrm{v}(\mathrm{C} 22 \mathrm{~N} 21 \mathrm{C} 18), \delta(\mathrm{CH})$ ring $\mathrm{C}$ \\
\hline 1270 & 1324 & 48 & - & - & - & $\delta(\mathrm{N} 17 \mathrm{H}), \delta(\mathrm{CH})$ ring $\mathrm{C}, v(\mathrm{CC})$ ring $\mathrm{C}, v(\mathrm{~N} 7 \mathrm{C} 11)$ \\
\hline- & - & - & 1277 & 1357 & 76 & $\delta(\mathrm{N} 7 \mathrm{H}), \delta(\mathrm{CH})$ ring $\mathrm{C}$ \\
\hline- & - & - & - & 1322 & 40 & $\delta(\mathrm{N} 21 \mathrm{H}), \delta(\mathrm{CH})$ ring $\mathrm{A}$ \\
\hline- & - & - & 1237 & 1299 & 124 & $\delta(\mathrm{N} 21 \mathrm{H}), \delta(\mathrm{N} 7 \mathrm{H}), \delta(\mathrm{CH})$ ring $\mathrm{A}, v(\mathrm{~N} 17 \mathrm{~N} 16)$ \\
\hline 1227 & 1294 & 24 & - & - & - & $\delta(\mathrm{N} 17 \mathrm{H}), \delta(\mathrm{N} 7 \mathrm{H}), \delta(\mathrm{CH})$ ring $\mathrm{C}, \nu(\mathrm{N} 7 \mathrm{C} 11)$ \\
\hline- & - & - & 1214 & 1269 & 163 & $\delta(\mathrm{CH})$ ring $\mathrm{A}, \delta(\mathrm{N} 7 \mathrm{H})$ \\
\hline- & - & - & - & 1251 & 15 & $\delta(\mathrm{N} 7 \mathrm{H}), \delta(\mathrm{CH})$ ring $\mathrm{A}, \delta(\mathrm{CH})$ ring $\mathrm{C}$ \\
\hline 1207 & 1246 & 56 & - & - & - & $\delta(\mathrm{N} 17 \mathrm{H}), \delta(\mathrm{N} 7 \mathrm{H}), \delta(\mathrm{CH})$ ring $\mathrm{C}$ \\
\hline 1198 & 1221 & 261 & - & - & - & $\delta(\mathrm{N} 17 \mathrm{H}), \delta(\mathrm{N} 7 \mathrm{H}), \delta(\mathrm{CH})$ ring $\mathrm{C}, v(\mathrm{C} 24 \mathrm{~F} 27)$ \\
\hline- & 1218 & 130 & - & - & - & $\delta(\mathrm{N} 17 \mathrm{H}), \delta(\mathrm{N} 7 \mathrm{H}), \delta(\mathrm{CH})$ ring $\mathrm{A}, \mathrm{v}(\mathrm{N} 17 \mathrm{C} 18), v(\mathrm{C} 24 \mathrm{~F} 27)$ \\
\hline- & - & - & 1169 & 1197 & 220 & $\delta(\mathrm{CH})$ ring $\mathrm{A}, \delta(\mathrm{CH})$ ring $\mathrm{C}, \delta(\mathrm{N} 7 \mathrm{H}), \delta(\mathrm{N} 21 \mathrm{H})$ \\
\hline 1134 & 1192 & 693 & - & - & - & $\delta(\mathrm{CH})$ ring $\mathrm{A}, \delta(\mathrm{N} 21 \mathrm{H}), \delta(\mathrm{CH})$ ring $\mathrm{C}, v(\mathrm{~N} 17 \mathrm{~N} 16), \delta($ ringB $)$ \\
\hline 1100 & 1179 & 69 & - & - & - & $\delta(\mathrm{CH})$ ring $\mathrm{C}, \delta(\mathrm{N} 7 \mathrm{H})$ \\
\hline- & - & - & - & 1180 & 39 & $\delta(\mathrm{CH})$ ring $\mathrm{C}$ \\
\hline- & - & - & - & 1177 & 48 & $\delta(\mathrm{CH})$ ring $\mathrm{A}$ \\
\hline- & - & - & 1143 & 1167 & 446 & $\delta(\mathrm{CH})$ ring $\mathrm{A}, \delta(\mathrm{CH})$ ring $\mathrm{C}, \delta(\mathrm{N} 7 \mathrm{H}), \delta(\mathrm{N} 21 \mathrm{H}), v(\mathrm{~N} 17 \mathrm{~N} 16)$ \\
\hline 1030 & 1163 & 147 & - & - & - & $\delta(\mathrm{C} 25 \mathrm{H}), \delta(\mathrm{C} 26 \mathrm{H}), \delta(\mathrm{C} 30 \mathrm{H}), \delta(\mathrm{N} 21 \mathrm{H}), \delta(\mathrm{N} 17 \mathrm{H}), \nu(\mathrm{N} 17 \mathrm{C} 18)$ \\
\hline 982 & 1123 & 12 & 1091 & 1126 & 99 & $\delta(\mathrm{CH})$ ring $\mathrm{C}$ \\
\hline 945 & 1112 & 6 & 1041 & 1116 & 83 & $\delta(\mathrm{CC})$ ring $\mathrm{A}, \delta(\mathrm{N} 21 \mathrm{H})$ \\
\hline 864 & 1056 & 14 & - & 1057 & 22 & $\delta(\mathrm{CH})$ ring $\mathrm{A}$, \\
\hline- & - & - & 1008 & 997 & 57 & $\omega(\mathrm{CH})$ ring $\mathrm{C}$ \\
\hline 817 & 963 & 46 & - & 832 & 150 & $v(\mathrm{~S} 20 \mathrm{C} 10)$ \\
\hline 788 & 832 & - & - & - & - & $v(\mathrm{C} 24 \mathrm{~F} 27), \delta$ (isatin) \\
\hline- & 808 & - & - & - & - & $\omega(\mathrm{CH})$ ring $\mathrm{C}, \omega(\mathrm{N} 17 \mathrm{H}), \omega(\mathrm{C} 11 \mathrm{O} 15)$ \\
\hline
\end{tabular}

of $\mathrm{NH}$ groups (H23 and H19) are seen at $\delta 11.26 \mathrm{ppm}$ and $\delta$ $12.86 \mathrm{ppm}$, respectively. The spectra for $\mathrm{Zn}\left[(\mathrm{I} 2 \mathrm{FPTH})_{2}\right]$ shows the presence of first peak at $\delta 11.03$ and the absence of second peak indicating that the hydrogen is deprotonated when the ligand coordinates through S20 and N16 atoms. C9(H14) proton signal for $\mathrm{I}_{2} \mathrm{FPTH}_{2}$ ligand seen at $\delta 11.26 \mathrm{ppm}$ shifts 


\begin{tabular}{|c|c|c|c|c|c|c|c|c|}
\hline \multicolumn{9}{|c|}{$\begin{array}{c}\text { TABLE-6 } \\
\text { I NMR CHEMICAL SHIFTS FOR I2FPTH, OBTAINED FROM RHF AND DFT METHODS }\end{array}$} \\
\hline \multicolumn{9}{|c|}{ RHF } \\
\hline $\mathrm{I}_{2} \mathrm{FPTH}_{2}$ & Exp. & $\begin{array}{c}\text { 3-21 } \\
\text { G(d.p) }\end{array}$ & $\begin{array}{c}3-21 G^{*} \\
\text { (d.p) }\end{array}$ & $\begin{array}{c}\text { 6-31 } \\
\text { G(d.p) }\end{array}$ & $\begin{array}{l}\text { 6-311 } \\
\text { G(d.p) }\end{array}$ & $\begin{array}{l}\text { 6-311+ } \\
\text { G(d.p) }\end{array}$ & $\begin{array}{c}\text { 6-311++ } \\
\text { G(d.p) }\end{array}$ & $\begin{array}{l}6-311++ \\
G(2 d .2 p)\end{array}$ \\
\hline H14 & 7.40 & 8.40 & 8.11 & 8.34 & 8.25 & 8.48 & 8.49 & 8.47 \\
\hline $\mathrm{H} 1$ & 6.94 & 7.00 & 7.05 & 7.08 & 6.95 & 7.10 & 7.09 & 7.06 \\
\hline $\mathrm{H} 10$ & 7.37 & 7.51 & 7.58 & 7.40 & 7.25 & 7.41 & 7.41 & 7.50 \\
\hline $\mathrm{H} 5$ & 7.49 & 7.88 & 7.75 & 7.80 & 7.68 & 7.82 & 7.81 & 7.91 \\
\hline $\mathrm{H} 29$ & 7.70 & 9.92 & 9.69 & 10.03 & 9.85 & 10.00 & 9.97 & 10.21 \\
\hline $\mathrm{H} 31$ & 7.32 & 7.25 & 7.17 & 7.51 & 7.39 & 7.55 & 7.54 & 7.62 \\
\hline $\mathrm{H} 32$ & 7.26 & 7.74 & 7.61 & 7.59 & 7.45 & 7.56 & 7.53 & 7.64 \\
\hline H33 & 7.10 & 7.52 & 7.47 & 7.47 & 7.29 & 7.40 & 7.39 & 7.51 \\
\hline $\mathrm{H} 23$ & 11.26 & 9.00 & 10.23 & 9.15 & 8.81 & 9.24 & 9.27 & 9.46 \\
\hline H19 & 12.86 & 11.41 & 12.91 & 11.32 & 10.95 & 11.41 & 11.41 & 11.75 \\
\hline $\mathrm{H} 12$ & 10.71 & 5.65 & 6.38 & 6.45 & 6.21 & 6.68 & 6.69 & 6.85 \\
\hline $\mathrm{R}^{2}$ & & 0.654 & 0.864 & 0.654 & 0.639 & 0.676 & 0.684 & 0.693 \\
\hline \multicolumn{9}{|c|}{ B3LYP } \\
\hline H14 & 7.40 & 7.43 & 7.24 & 7.74 & 7.90 & 8.03 & 8.01 & 8.06 \\
\hline $\mathrm{H} 1$ & 6.94 & 6.38 & 6.18 & 6.72 & 6.86 & 6.87 & 6.83 & 7.00 \\
\hline $\mathrm{H} 10$ & 7.37 & 6.90 & 6.71 & 7.15 & 7.25 & 7.32 & 7.31 & 7.41 \\
\hline $\mathrm{H} 5$ & 7.49 & 7.08 & 6.89 & 7.32 & 7.42 & 7.51 & 7.49 & 7.62 \\
\hline $\mathrm{H} 29$ & 7.70 & 9.14 & 8.94 & 9.84 & 10.00 & 10.06 & 10.03 & 10.36 \\
\hline $\mathrm{H} 31$ & 7.32 & 6.49 & 6.29 & 7.09 & 7.26 & 7.33 & 7.31 & 7.46 \\
\hline $\mathrm{H} 32$ & 7.26 & 6.96 & 6.75 & 7.24 & 7.35 & 7.30 & 7.33 & 7.46 \\
\hline $\mathrm{H} 33$ & 7.10 & 6.82 & 6.61 & 7.13 & 7.19 & 7.16 & 7.16 & 7.31 \\
\hline $\mathrm{H} 22$ & 11.26 & 9.57 & 9.35 & 10.04 & 9.98 & 10.26 & 10.30 & 10.60 \\
\hline H19 & 12.86 & 12.44 & 12.22 & 12.71 & 12.50 & 12.78 & 12.76 & 13.27 \\
\hline H12(indole) & 10.71 & 5.71 & 5.51 & 6.51 & 6.54 & 6.82 & 6.85 & 7.07 \\
\hline$R^{2}$ & & 0.852 & 0.854 & 0.831 & 0.808 & 0.825 & 0.830 & 0.830 \\
\hline
\end{tabular}
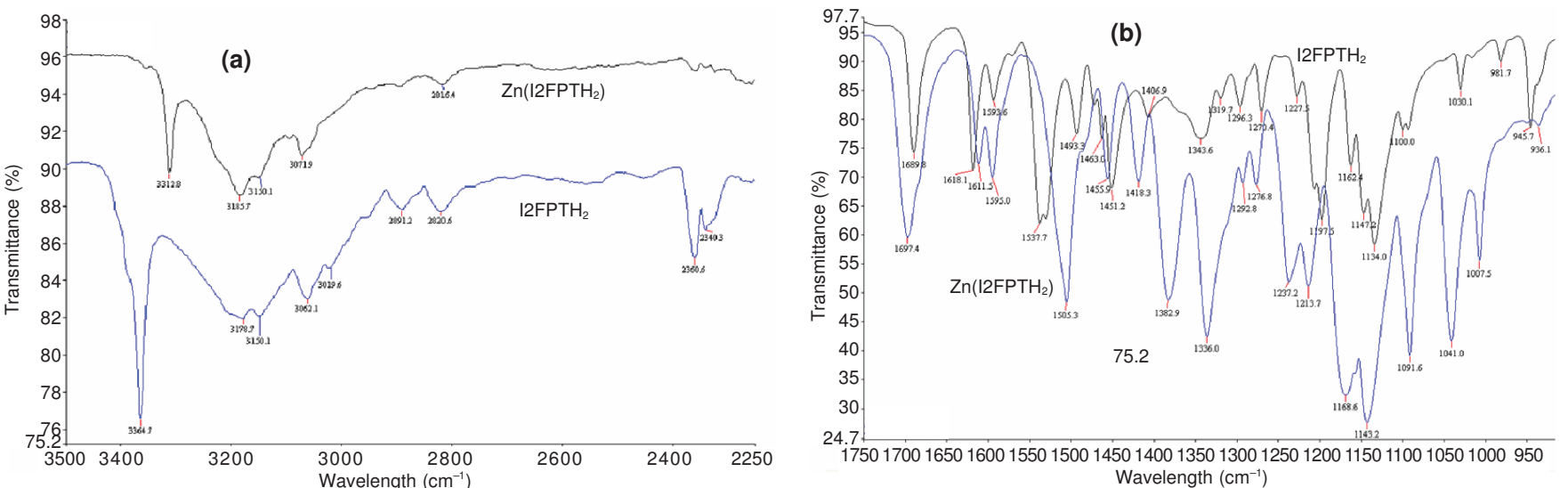
Wavelength $\left(\mathrm{cm}^{-1}\right)$

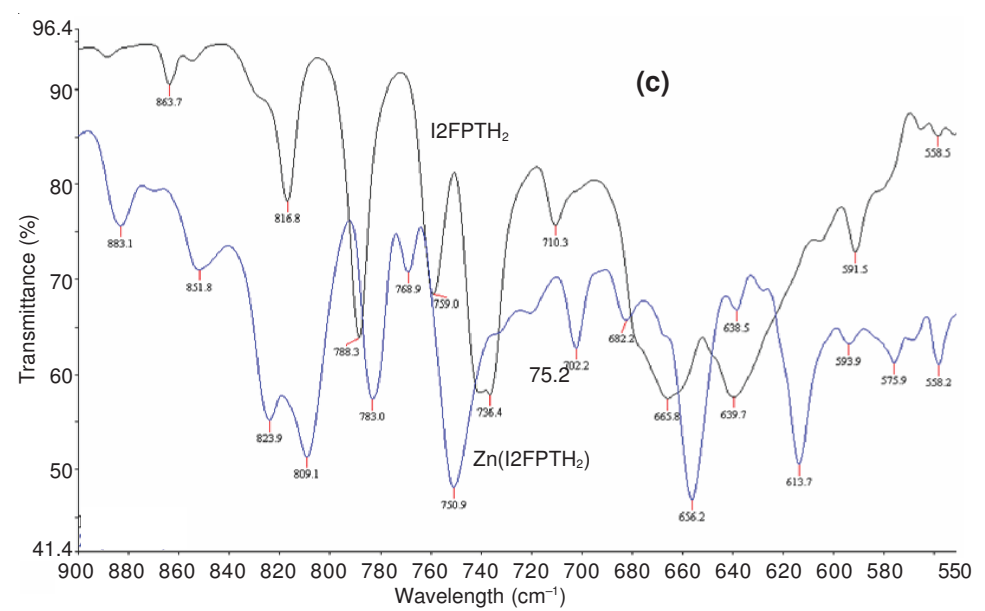

Fig. 3. (a-c) Experimental FT-IR graphics of 4-(2-fluorophenyl)-1-(2-oxoindolin-3-ylidene)thiosemicarbazone "I2FPTH ${ }_{2}$ " and its zinc(II) complex " $\mathrm{Zn}\left(\mathrm{I}_{2} \mathrm{FPTH}_{2}\right)$ " 
Vol. 25, No. 17 (2013) Synthesis and Quantum Chemical Calculations of 4-(2-Fluorophenyl)-1-(2-oxoindolin-3-ylidene)thiosemicarbazone 9729

\begin{tabular}{|c|c|c|c|c|c|c|c|c|}
\hline \multicolumn{9}{|c|}{$\begin{array}{l}\text { TABLE-7 } \\
\text { TH }_{2} \text { OBTA }\end{array}$} \\
\hline \multicolumn{9}{|c|}{ RHF } \\
\hline $\mathrm{I}_{2} \mathrm{FPTH}_{2}$ & Exp. & $\begin{array}{c}3-21 \mathrm{G} \\
(\mathrm{d}, \mathrm{p})\end{array}$ & $\begin{array}{c}3-21 \mathrm{G}^{*} \\
(\mathrm{~d}, \mathrm{p})\end{array}$ & $\begin{array}{c}6-31 \\
G(d, p) \\
\end{array}$ & $\begin{array}{l}6-311 \\
G(d, p) \\
\end{array}$ & $\begin{array}{l}6-311+ \\
G(d, p)\end{array}$ & $\begin{array}{c}\text { 6-311++ } \\
\text { G(d,p) }\end{array}$ & $\begin{array}{c}6-311++2 \\
G(2 d, 2 p) \\
\end{array}$ \\
\hline C9 & 130 & 119 & 119 & 126 & 132 & 133 & 133 & 131 \\
\hline $\mathrm{C} 5$ & 122 & 115 & 121 & 121 & 127 & 128 & 128 & 126 \\
\hline $\mathrm{C} 3$ & 132 & 127 & 126 & 134 & 141 & 142 & 142 & 141 \\
\hline $\mathrm{C} 2$ & 116 & 104 & 109 & 108 & 114 & 114 & 114 & 113 \\
\hline $\mathrm{C} 4$ & 143 & 134 & 136 & 143 & 150 & 151 & 151 & 150 \\
\hline $\mathrm{C} 8$ & 120 & 110 & 118 & 117 & 123 & 124 & 124 & 123 \\
\hline $\mathrm{C} 18$ & 133 & 128 & 128 & 129 & 133 & 134 & 134 & 134 \\
\hline $\mathrm{C} 11$ & 163 & 161 & 160 & 161 & 166 & 168 & 168 & 168 \\
\hline $\mathrm{C} 18$ & 178 & 188 & 164 & 192 & 197 & 199 & 199 & 197 \\
\hline $\mathrm{C} 22$ & 131 & 122 & 127 & 127 & 133 & 134 & 134 & 133 \\
\hline $\mathrm{C} 25$ & 158 & 141 & 150 & 148 & 155 & 156 & 156 & 155 \\
\hline $\mathrm{C} 24$ & 123 & 115 & 116 & 122 & 128 & 128 & 128 & 127 \\
\hline $\mathrm{C} 26$ & 112 & 110 & 113 & 114 & 120 & 121 & 121 & 119 \\
\hline $\mathrm{C} 28$ & 127 & 119 & 122 & 124 & 131 & 132 & 132 & 130 \\
\hline C30 & 125 & 117 & 120 & 124 & 130 & 131 & 131 & 129 \\
\hline $\mathrm{R}^{2}$ & - & 0.872 & 0.967 & 0.947 & 0.946 & 0.946 & 0.946 & 0.953 \\
\hline \multicolumn{9}{|c|}{ B3LYP } \\
\hline C9 & 129.50 & 108.53 & 107.48 & 116.43 & 126.60 & 126.83 & 126.73 & 126.05 \\
\hline C5 & 121.71 & 110.19 & 109.16 & 118.20 & 128.48 & 129.06 & 129.51 & 128.21 \\
\hline C3 & 132.01 & 116.11 & 115.03 & 125.01 & 136.09 & 136.62 & 136.57 & 135.79 \\
\hline $\mathrm{C} 2$ & 116.45 & 98.59 & 97.56 & 105.46 & 114.20 & 114.68 & 114.62 & 113.69 \\
\hline $\mathrm{C} 4$ & 143.09 & 126.01 & 124.98 & 136.55 & 148.03 & 148.49 & 148.62 & 148.17 \\
\hline $\mathrm{C} 8$ & 120.32 & 107.90 & 106.92 & 117.60 & 127.26 & 128.17 & 128.35 & 127.94 \\
\hline $\mathrm{C} 13$ & 133.19 & 118.10 & 116.96 & 123.64 & 131.59 & 132.59 & 132.36 & 131.88 \\
\hline $\mathrm{C} 11$ & 163.11 & 149.03 & 147.97 & 155.82 & 166.26 & 168.87 & 168.88 & 168.88 \\
\hline $\mathrm{C} 18$ & 178.41 & 160.59 & 158.11 & 169.80 & 180.64 & 182.41 & 182.52 & 181.59 \\
\hline $\mathrm{C} 22$ & 130.61 & 116.27 & 115.38 & 124.26 & 134.65 & 135.41 & 135.30 & 134.88 \\
\hline $\mathrm{C} 25$ & 157.74 & 140.15 & 139.11 & 148.48 & 161.87 & 162.28 & 162.61 & 162.08 \\
\hline $\mathrm{C} 24$ & 122.90 & 105.64 & 104.59 & 114.46 & 124.12 & 123.85 & 124.62 & 124.42 \\
\hline $\mathrm{C} 26$ & 111.61 & 102.31 & 101.26 & 109.52 & 119.18 & 119.52 & 119.83 & 118.86 \\
\hline $\mathrm{C} 28$ & 126.94 & 111.45 & 110.39 & 119.39 & 129.99 & 130.52 & 130.84 & 129.66 \\
\hline C30 & 124.87 & 110.35 & 109.25 & 118.67 & 129.14 & 129.32 & 129.46 & 128.78 \\
\hline $\mathrm{R}^{2}$ & & 0.872 & 0.967 & 0.947 & 0.970 & 0.970 & 0.968 & 0.969 \\
\hline
\end{tabular}

TABLE-8

${ }^{1} \mathrm{H}$ AND ${ }^{13} \mathrm{C}$ NMR CHEMICAL SHIFT FOR Zn[(I2FPTH $\left.)_{2}\right]$

\begin{tabular}{|c|c|c|c|c|c|c|c|}
\hline \multicolumn{8}{|c|}{${ }^{1} \mathrm{H}$} \\
\hline Atom & Exp. & 6-311 G(d,p) & $6-311+G(d, p)$ & Atom & Exp & 6-311 G(d,p) & $6-311+G(d, p)$ \\
\hline H14 & 7.70 & 7.73 & 8.60 & H31 & 7.70 & 7.23 & 7.22 \\
\hline $\mathrm{H} 1$ & 7.10 & 6.76 & 6.93 & $\mathrm{H} 32$ & 7.36 & 7.53 & 7.58 \\
\hline $\mathrm{H} 10$ & 7.26 & 7.21 & 7.25 & $\mathrm{H} 33$ & 7.26 & 7.17 & 7.17 \\
\hline H5 & 7.01 & 6.60 & 6.4 & $\mathrm{H} 22$ & 11.03 & 7.95 & 7.87 \\
\hline $\mathrm{H} 29$ & 8.00 & 10.35 & 10.54 & $\mathrm{H} 12$ & 10.67 & 6.16 & 6.28 \\
\hline \multicolumn{8}{|l|}{${ }^{13} \mathrm{C}$} \\
\hline C9 & - & 130.08 & 131.47 & $\mathrm{C} 18$ & - & 182.80 & 184.29 \\
\hline $\mathrm{C} 5$ & - & 126.00 & 127.31 & $\mathrm{C} 22$ & - & 133.99 & 134.36 \\
\hline $\mathrm{C} 3$ & - & 137.79 & 138.42 & $\mathrm{C} 25$ & - & 160.95 & 161.51 \\
\hline $\mathrm{C} 2$ & - & 112.54 & 112.75 & $\mathrm{C} 24$ & - & 130.43 & 131.59 \\
\hline $\mathrm{C} 4$ & - & 147.39 & 148.14 & $\mathrm{C} 26$ & - & 118.35 & 118.80 \\
\hline $\mathrm{C} 8$ & - & 124.74 & 126.32 & $\mathrm{C} 28$ & - & 131.44 & 132.12 \\
\hline $\mathrm{C} 13$ & - & 148.69 & 151.23 & $\mathrm{C} 30$ & - & 128.80 & 129.33 \\
\hline $\mathrm{C} 11$ & 166.79 & 161.72 & 163.97 & - & - & - & - \\
\hline
\end{tabular}

downfield to $7.70 \mathrm{ppm}$ in $\mathrm{Zn}\left[(\mathrm{I} 2 \mathrm{FPTH})_{2}\right]$ complex due to the nearby position to $\mathrm{C}=\mathrm{N}$ group and may be the most affected by the $\mathrm{Zn}-\mathrm{N}$ bond formation ${ }^{19}$.

UV study: The UV visible spectral values, excitation energies and oscillator strengths for $\mathrm{I}_{2} \mathrm{FPTH}_{2}$ ligand were performed at the TDB3LYP levels by using 6-311G(d,p), 6$311++\mathrm{G}(\mathrm{d}, \mathrm{p})$ and 6-311G(d,p) basis sets for $\mathrm{Zn}\left[(\mathrm{I} 2 \mathrm{FPTH})_{2}\right]$ complex, as shown in Table-9. The bands assigned experimentally at $256 \mathrm{~nm}$ were found at $256 \mathrm{~nm}$ with the calculation TDB3LYP/6-311G(d,p) due to transitions mainly from HOMO-2 to LUMO+1 and from HOMO to LUMO+2. HOMO-2 and HOMO consist of $-7.5 \% 2 \mathrm{pz}(\mathrm{N} 21)+7.5 \%$ $2 \mathrm{pz}(\mathrm{N} 17)-6.2 \% 2 \mathrm{pz}(\mathrm{C} 30)+5.0 \% 2 \mathrm{pz}(\mathrm{C} 22)-4.5 \% 3 \mathrm{pz}(\mathrm{C} 30)+$ $4.5 \% 2 \mathrm{pz}(\mathrm{C} 24)$ and $+30.9 \% 3 \mathrm{pz}(\mathrm{S} 20)+17.9 \% 4 \mathrm{pz}(\mathrm{S} 20)-$ 


\begin{tabular}{|c|c|c|}
\hline \multicolumn{3}{|c|}{$\begin{array}{l}\text { UV VISIBLE SPECTRAL VALUES, EXCITATION } \\
\text { ENERGIES AND OSCILLATOR STRENGTHS FOR I2FPTH } \\
\left.\text { LIGAND AND Zn[(I2FPTH })_{2}\right](\mathrm{nm}) \\
\text { Lit) }\end{array}$} \\
\hline \multirow{2}{*}{ Experiment } & \multicolumn{2}{|c|}{$\mathrm{I}_{2} \mathrm{FPTH}_{2}$} \\
\hline & $370 \mathrm{~nm}(27027)$ & $256 \mathrm{~nm}(39062)$ \\
\hline 6-311 G(d,p) & $352[3.51(0.46) \mathrm{E} 4]$ & $256[4.85(0.18) \mathrm{E} 10]$ \\
\hline $6-311+\mathrm{G}(\mathrm{d}, \mathrm{p})$ & $359[3.45(0.45) \mathrm{E} 4]$ & $267[4.64(0.16) \mathrm{E} 9]$ \\
\hline $6-311++\mathrm{G}(\mathrm{d}, \mathrm{p})$ & $359[3.45(0.45) \mathrm{E} 4]$ & $267[4.64(0.16) \mathrm{E} 9]$ \\
\hline \multirow{2}{*}{ Experiment } & \multicolumn{2}{|c|}{$\mathrm{Zn}\left[(\mathrm{I} 2 \mathrm{FPTH})_{2}\right]$} \\
\hline & $435 \mathrm{~nm}$ & $252 \mathrm{~nm}$ \\
\hline 6-311 G(d,p) & $462[2.68(0.24) \mathrm{E} 5]$ & $264[4.69(0.32) \mathrm{E} 32]$ \\
\hline $6-311+\mathrm{G}(\mathrm{d}, \mathrm{p})$ & $479[2.59(0.25) \mathrm{E} 3]$ & $267[4.64(0.30) \mathrm{E} 32]$ \\
\hline
\end{tabular}

$5.4 \% 2 \mathrm{pz}(\mathrm{N} 21)-3.4 \%$ 2pz(N17)-2.9 \% 3pz(N21)- $2.9 \%$ $2 \mathrm{pz}(\mathrm{C} 30)$. LUMO+1 and LUMO+2 consist of $+14.1 \%$ 2pz(C18)- 9.1 \% 2pz(C30)- $8.3 \%$ 2pz(C22)-7.3\% 3pz(C30)+ $6.7 \% 3 \mathrm{pz}(\mathrm{C} 18)-6.3 \% 2 \mathrm{pz}(\mathrm{N} 17)$ and $+15.9 \% 2 \mathrm{pz}(\mathrm{C} 2)+$ $15.6 \% 2 \mathrm{pz}(\mathrm{C} 9)+12.7 \% 3 \mathrm{pz}(\mathrm{C} 2)+12.2 \% 3 \mathrm{pz}(\mathrm{C} 9)+6.1 \%$ $2 \mathrm{pz}(\mathrm{C} 11)-6.0 \% 2 \mathrm{pz}(\mathrm{C} 4)$

The bands assigned experimentally at $370 \mathrm{~nm}$ were calculated with the calculation TDB3LYP/6-311G(d,p) as $352 \mathrm{~nm}$ due to transitions mainly due to from HOMO-3 to LUMO consisting of $+17.5 \% 2 \mathrm{pz}(\mathrm{C} 24)+14.1 \% 2 \mathrm{pz}(\mathrm{C} 28)+$ $11.5 \% 3 \mathrm{pz}(\mathrm{C} 28)-11.3 \%$ 2pz(C25)- $11.1 \%$ 2pz(C26)- $9.1 \%$ $3 \mathrm{pz}(\mathrm{C} 26)$ and $+16.5 \% 2 \mathrm{pz}(\mathrm{N} 16)+8.3 \% 3 \mathrm{pz}(\mathrm{N} 16)-7.1 \%$ $2 \mathrm{pz}(\mathrm{C} 13)+5.8 \% 2 \mathrm{pz}(\mathrm{O} 15)-5.7 \% 3 \mathrm{pz}(\mathrm{C} 13)-5.6 \% 2 \mathrm{pz}(\mathrm{C} 11)$.

The bands of $\mathrm{I}_{2} \mathrm{FPTH}_{2}$ ligand at 370 and $256 \mathrm{~nm}$ shifts to 435 and $252 \mathrm{~nm}$ in the spectrum of $\mathrm{Zn}\left[(\mathrm{I} 2 \mathrm{FPTH})_{2}\right]$. This changes are due to the electron delocalization on thiosemicarbazone group upon coordination with $\mathrm{Zn}(\mathrm{II})$ ions.

\section{Conclusion}

The optimized geometrical parameters, bond lengths and bond angles, were calculated theoretically by using the density functional and RHF methods and compared with the experimental data for studied molecule. Using of RHF method is somewhat better than the B3LYP for all calculated basis sets for the calculations of bond lengths. The highest correlation coefficient for bond lengths was 0.9745 for RHF/6-311++ $\mathrm{G}(2 \mathrm{~d}, 2 \mathrm{p})$. The agreement between the calculated and the experimental bond angles is better than for the bond lengths.

Moreover, according to results of Fukui functions values of $\mathrm{I}_{2} \mathrm{FPTH}_{2}$, it is found that sulphur atom contributes dominantly to the HOMO, while nitrogen 16 atom play important role for contribution to the LUMO.

The IR spectrum of $\mathrm{I}^{2} \mathrm{FPTH}_{2}$ shows that bands at 3313 $\mathrm{cm}^{-1}$ attributed to $\mathrm{v}(\mathrm{NH}) \mathrm{cm}^{-1}$ vibration of the indole ring and thiosemicarbazone moiety. It is also concluded from IR results that a very strong band at 1690 and $1697 \mathrm{~cm}^{-1}$ assigned to $(\mathrm{C}=\mathrm{O})$ stretching vibration of ligand and its $\mathrm{Zn}$ (II) complex. Besides, the band corresponding to the stretching vibration of the $\mathrm{C}=\mathrm{S}$ group appears at $1200-1194 \mathrm{~cm}^{-1}$.

According to results of the theoretical studies of ${ }^{1} \mathrm{H}$ and ${ }^{13} \mathrm{C}$ NMR chemical shifts for $\mathrm{I}_{2} \mathrm{FPTH}_{2}$, since $\mathrm{C} 9(\mathrm{H} 14)$ proton signal seen at $\delta 11.26 \mathrm{ppm}$ shifts downfield to $7.70 \mathrm{ppm}$ in $\mathrm{Zn}\left[(\mathrm{I} 2 \mathrm{FPTH})_{2}\right]$ complex due to the nearby position to $\mathrm{C}=\mathrm{N}$ group, it can be said that the $\mathrm{Zn}-\mathrm{N}$ bond formation effects this proton signal.
In addition, the bands assigned at $256 \mathrm{~nm}$ were found at $256 \mathrm{~nm}$ with the calculation TDB3LYP/6-311G(d,p) due to transitions mainly from HOMO-2 to LUMO+1 and from $\mathrm{HOMO}$ to $\mathrm{LUMO}+2$ due to the results of calculation of UV visible spectral values, excitation energies and oscillator strengths for $\mathrm{I}^{2} \mathrm{FPTH}_{2}$ ligand.

In this study, results obtained theoretically are sensibly consistent with the results of experimental study.

\section{ACKNOWLEDGEMENTS}

The financial support for this study was provided by the BAP Fund (Project Number: FEB 2010/37)

\section{REFERENCES}

1. S.K. Sridhar and R. Atmakuru, Indian Drugs, 38, 174 (2001).

2. S.N. Pandeya and D. Sriram, Acta Pharm. Turc., 40, 33 (1998).

3. R.S. Varma and W.L. Nobles, J. Pharm. Sci., 64, 881 (1975).

4. S.N. Pandeya, P. Yogeeswari, D. Sriram, E. De Clercq, C. Pannecouque and M. Witvrouw, Chemotherapy, 45, 192 (1999).

5. C. David, T. Marie and G. Roussel, U.S. Patent, 5,498,716 (1996); Chem. Abstr., 124, 343271 (1996).

6. S.N. Pandeya, D. Sriram, G. Nath and Clercq E. De, Pharm. Acta Helv., 74, 11 (1999).

7. S.A. Imam and R.S. Varma, Experientia, 31, 1287 (1975).

8. N. Karali, A. Gürsoy, F. Kandemirli, N. Shvets, F.B. Kaynak, S. Özbey, V. Kovalishyn and A. Dimoglo, Bioorg. Med. Chem., 15, 5888 (2007).

9. G. Cerchiaro and A.C. Ferreira, J. Braz. Chem. Soc., 17, 1473 (2006).

10. S. Gunesdogdu-Sagdinc, B. Köksoy, F. Kandemirli and S.H. Bayari, J. Mol. Struct., 917, 63 (2009).

11. F. Kandemirli, T. Arslan, N. Karadayi, E.E. Ebenso and B. Köksoy, J. Mol. Struct., 938, 89 (2009).

12. F. Kandemirli, T. Arslan, B. Koksoy and M. Yilmaz, J. Chem. Soc. Pak., 31, 498 (2009).

13. F. Kandemirli, B. Koksoy, T. Arslan, S. Sagdinc and H. Berber, J. Mol. Struct., 921, 172 (2009).

14. H. Pervez, M. Yaqub, M. Ramzan, M.S. Iqbal and M.N. Tahir, Acta Cryst., E66, 01405 (2010).

15. S.G. Sagdinc, F. Kandemirli, B. Köksoy and S.H. Bayari, Phosphorus Sulfur Silicon Rel. Elem., 187, 1243 (2012),

16. M.J. Frisch, G.W. Trucks, H.B. Schlegel, G.E. Scuseria, M.A. Robb, J.R. Cheeseman, G. Scalmani, V. Barone, B. Mennucci, G.A. Petersson, H. Nakatsuji, M. Caricato, X. Li, H.P. Hratchian, A.F. Izmaylov, J. Bloino, G. Zheng, J.L. Sonnenberg, M. Hada, M. Ehara, K. Toyota, R. Fukuda, J. Hasegawa, M. Ishida, T. Nakajima, Y. Honda, O. Kitao, H. Nakai, T. Vreven, J.A. Montgomery Jr., J.E. Peralta, F. Ogliaro, M. Bearpark, J.J. Heyd, E. Brothers, K.N. Kudin, V.N. Staroverov, T. Keith, R. Kobayashi, J. Normand, K. Raghavachari, A. Rendell, J.C. Burant, S.S. Iyengar, J. Tomasi, M. Cossi, N. Rega, J.M. Millam, M. Klene, J.E. Knox, J.B. Cross, V. Bakken, C. Adamo, J. Jaramillo, R. Gomperts, R.E. Stratmann, O. Yazyev, A.J. Austin, R. Cammi, C. Pomelli, J.W. Ochterski, R.L. Martin, K. Morokuma, V.G. Zakrzewski, G.A. Voth, P. Salvador, J.J. Dannenberg, S. Dapprich, A.D. Daniels, O. Farkas, J.B. Foresman, J.V. Ortiz, J. Cioslowski and D.J. Fox, Gaussian 09, Revision B. 01, Gaussian, Inc., Wallingford CT (2010).

17. S.I. Gorelsky, AOMix: Program for Molecular Orbital Analysis; University of Ottawa, (2009); http://www.sg-chem.net/

18. S.I. Gorelsky and A.B.P. Lever, J. Organomet. Chem., 635, 187 (2001).

19. N.T. Akinchan, P.M. Drozdzewski and W. Holzer, J. Mol. Struct., 641, 17 (2002).

20. A.M. Asiri, A. Asiri and S.A. Khan, Molecules, 15, 4784 (2010). 